\title{
Viral suppression of RNA silencing
}

\author{
JIANG Lin, WEI ChunHong \& LI Yi \\ State Key Laboratory of Protein and Plant Gene Research, College of Life Sciences, Peking University, Beijing 100871, China
}

Received November 30, 2011; accepted December 7, 2011

\begin{abstract}
Gene silencing (RNA silencing) plays a fundamental role in antiviral defense in plants, fungi and invertebrates. Viruses encode proteins that suppress gene silencing to counter host defense. Viral suppressors of RNA silencing (VSRs) have been identified from almost all plant virus genera and some viruses of insects and mammals. Recent studies have revealed that VSRs counter host defense and interfere with host gene regulation by interacting with RNA or important components of the RNA silencing pathway. Here, we review the current understanding of the complex mechanisms of VSRs that have been revealed by recent studies.
\end{abstract}

RNA silencing, viral suppressor of RNA silencing, mechanisms

Citation: Jiang L, Wei C H, Li Y. Viral suppression of RNA silencing. Sci China Life Sci, 2012, 55: 109-118, doi: 10.1007/s11427-012-4279-x

\section{RNA silencing and RNA-based virus immu- nity}

In most eukaryotes, including plants, fungi, invertebrates and mammals, gene silencing (RNA silencing) refers to the mechanism of gene expression regulation by several classes of small RNAs in a sequence-specific manner. RNA silencing plays a fundamental role in regulating development, maintaining genome stability and responding to environmental stress. As RNA silencing also contributes to antiviral immunity in plants, fungi and invertebrates, it is an important part of innate immunity. When RNA silencing is used to defend against viruses, it is also referred to as RNA-based virus immunity (RVI) [1].

The key molecules in gene silencing are small RNAs, including siRNA, miRNA and piRNA [2]. siRNA and miRNA are both produced by Dicer or Dicer like (DCL), an RNase III nuclease. In general, siRNAs are produced from long dsRNA, while the precursor of miRNA is hairpin-like RNA (hpRNA) [3]. Small RNA molecules are loaded onto

*Corresponding author (email: liyi@pku.edu.cn)
RNA-induced silencing complexes (RISC), which are composed of Argonaute (AGO) and other related proteins, which guide cleavage of the target RNA, inhibition of translation of the target mRNA or genome DNA methylation [4]. To amplify siRNAs, RNA-dependent RNA polymerases (RDRs) convert single stranded RNA into dsRNA to produce secondary siRNA [5].

The pathway of RVI can be divided into four major steps: (i) Viral RNAs are sensed and processed into virus-derived siRNA (vsiRNA) by Dicer; (ii) RDRs and other proteins participate in the amplification of vsiRNA; (iii) vsiRNAs are assembled into RISC to degrade viral RNAs or mediate the methylation of viral DNA; and (iv) systemic RNA silencing and systemic antiviral defense are established [6].

Four DCL genes, six RDR genes and 10 AGO genes were identified in the model plant Arabidopsis thaliana. Among the four DCL genes, DCL4 and DCL2 are the most important in producing vsiRNA in a hierarchical manner, producing 21-nt and 22-nt vsiRNA molecules, respectively. DCL4 is the primary gene responsible for producing 21-nt vsiRNA, while DCL2 processes viral RNAs into 22-nt vsiRNA when the function of DCL4 is inhibited or lost. 
DCL3 produces 24-nt vsiRNA [7-10]. Among the six RDR genes, RDR1 and RDR6 have been established as participating in antiviral defense [8,11-13]. RDR1 and RDR6 both participate in the amplification of secondary vsiRNA, but their respective vsiRNA products are derived from different viral genomic regions. Thus, it has been speculated that the substrates recognized by RDR1 and RDR6 are different. It is possible that RDR 1 recognizes the aberrant viral RNA molecules that are derived during virus replication, while RDR6 molecules could be recruited to viral mRNAs [14,15]. AGO1 and AGO2 participate in antiviral defense in a cooperative manner $[14,16]$, as both have the ability to bind vsiRNA. However, the two AGOs select vsiRNAs according to their length and the 5' terminal nucleotide. AGO1 preferentially binds to 21-nt vsiRNA with $\mathrm{U}$, while AGO2 preferentially binds to vsiRNA with $A$, as the $5^{\prime}$ terminal nucleotide [17].

The viral proteins known as VSRs have the ability to inhibit RNA silencing. In 1998, the HC-Pro and 2b proteins encoded by tobacco etch virus (TEV) and cucumber mosaic virus (CMV), respectively, were the first VSRs identified [18-20]. Currently, VSRs have been identified in almost all plant virus genera and in some insect and mammalian viruses, but have not exhibited any similarity in sequence or structure [21]. Recent studies have revealed that VSRs interact with multiple components of the RNA silencing pathway, inhibiting RNA silencing through multiple mechanisms (Figure 1).

\section{Mechanisms of viral suppressors of RNA si- lencing}

\subsection{VSRs target RNA components of RNA silencing}

A common strategy by which many identified VSRs interfere with the RNA silencing pathway is to bind long dsRNA or siRNA. Table 1 shows a group of VSRs that have the ability to bind dsRNA without size selection. B2, the VSR encoded by flock house virus (FHV), contains a dsRNA binding domain and binds various lengths of dsRNA in a dimer form. B2 inhibits the processing of long dsRNA into siRNA in vitro [22] and suppresses RNA silencing when it is expressed in cells prior to the introduction of long dsRNA. B2 interacts with viral dsRNA replication intermediates (vRI-dsRNA) in the FHV-infected Drosophila cells and represses the accumulation of vsiRNA during FHV infection. FHV with deficient B2 cannot accumulate in wild type Drosophila cells, but this accumulation is rescued in Drosophila cells with a Dicer-1 mutation. These results suggest that B2 interacts with vRI-dsRNA to protect the viral RNAs from being processed by Dicer [23]. P14, which is encoded by the Pothos latent aureusvirus (PoLV), also has the ability to bind dsRNA without size selection. Although P14 suppresses the accumulation of vsiRNA, the accumulation of viral genomic RNAs is not affected by the absence of P14, implying that the function of P14 is different from B2. Leaves infected systemically with the P14-deletion mutant of PoLV recover from the symptoms of infection, suggesting that P14 might inhibit systemic antiviral defense [24]. The $2 \mathrm{~b}$ proteins encoded by tomato aspermy virus (TAV) and CMV have the ability in vitro to bind dsRNA of 20-30 bp $[25,26]$. TAV 2 b constitutes two $\alpha$-helix structures and forms homodimers. The dimer recognizes two $\alpha$-helical turns of an A-form RNA duplex using a pair of hook-like structures that comprises four $\alpha$-helix structures. The TAV $2 \mathrm{~b}$ dimer further oligomerizes to form a tetramer [27].

Crystal studies of P19 encoded by carnation Italian ringspot virus (CIRV) and tomato bushy stunt virus (TBSV) have shown that P19 forms a homodimer and specifically binds siRNA within the duplex region. P19 has a high affinity for 19-bp dsRNA with blunt ends or a 2-nucleotide 3' overhang [28-30]. In a Drosophila in vitro system, P19 prevents siRNA incorporation into RISC, but it does not prevent the assembled RISC from degrading the target RNA. The binding of P19 to vsiRNA has been confirmed by immunoprecipitation and gel filtration assays. However, both P19-deficient and wild type cymbidium ringspot virus (CymRSV) accumulate at similar levels in both protoplasts and inoculated leaves, indicating that P19 does not prevent RISC from degrading viral RNAs by sequestering vsiRNA [31,32]. In the systemic leaves, the P19-deficient CymRSV accumulates only in the vascular bundles and exhibits defects in invading the surrounding tissues [33]. Recent studies have shown that P19 specifically sequesters the DCL4-dependent 21-nt siRNAs derived from transgene RNAs, which move into the neighboring recipient cells and act as a silencing signal [34]. These results imply that P19 promotes systemic virus infection by sequestering the vsiRNA, preventing the signal for RNA silencing from spreading out of vascular bundles into neighboring cells.

Other VSRs bind to dsRNA in a size-selective manner, such as HC-Pro which is encoded by TEV, P21 which is encoded by beet yellows virus (BYV) and P122 which is encoded by tobacco mosaic virus (TMV). These VSRs preferentially bind to siRNA/miRNA with a 2-nt 3' overhang. They also inhibit the incorporation of siRNA/miRNA into RISC in vitro but do not interfere with programmed RISC activity [32,35]. P122 and HC-Pro have been shown to suppress 3 '-end methylation of miRNA and siRNA, respectively, possibly by competing with HEN1 methyltransferase for binding to miRNA/siRNA and thus interfering with the incorporation into RISC [35-37].

Sweet potato chlorotic stunt virus (SPCSV) encodes an RNA endoribonuclease III (RNase 3) that suppresses RNA silencing through a novel mechanism [38,39]. RNase 3 consists of an RNase domain and a dsRNA binding domain and cleaves siRNAs of 21-24 bp into 14-bp fragments in vitro, which are inactive in RNA silencing. The expression of RNase 3 inhibits the accumulation of siRNA originating from the host, but does not significantly change the siRNA 


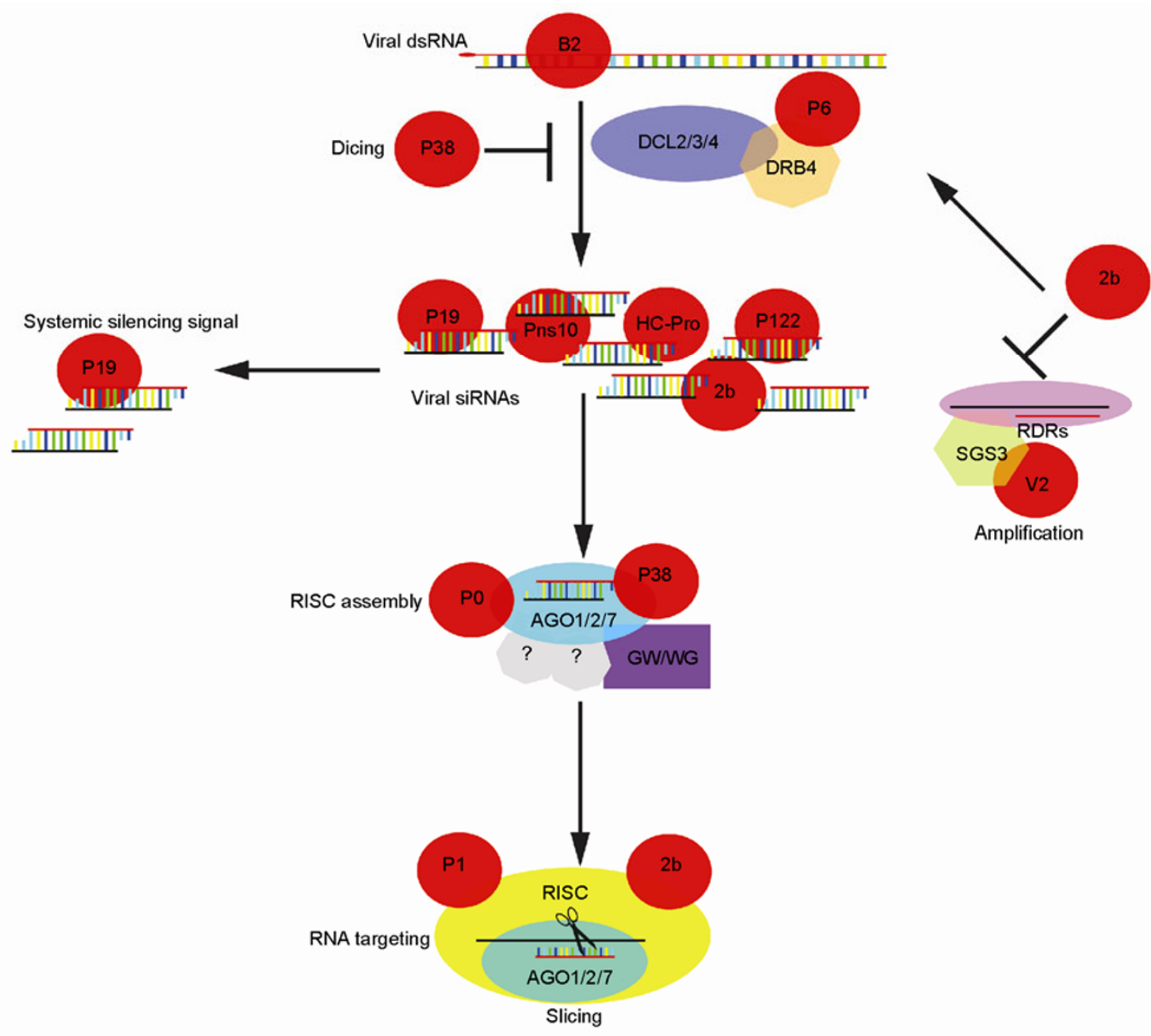

Figure 1 Schematic illustrating suppression of antiviral RNA silencing in plants by VSRs. Viral dsRNAs or hairpin-like RNAs are sensed and diced into siRNA by RNase III molecules, which are called DCLs (DCL2/3/4). The virus-derived siRNA is assembled into RISC by AGOs (AGO1/2/7) and other related proteins, some of which contain GW/WG motifs that interact with AGOs. The mature vsiRNA-loaded RISC targets viral RNA by slicing. Plant RDRs and their cofactors (SGS3) participate in the amplification of viral secondary siRNA. Viral siRNA is also mobile and induces systemic RNA silencing. VSRs prevent the assembly of different effectors or inhibit their function to disrupt the pathway at multiple steps.

derived from the virus. These data suggest that RNase 3 cleaves the siRNA that originates from the host, but not that derived from the virus itself [39]. However, long dsRNA is another possible target for RNase 3 [21].

\subsection{VSRs target AGO proteins}

AGO proteins that bind small RNAs to assemble the active RISC are important effectors in RNA silencing. This family of proteins consists of PAZ, MID and PIWI domains. The PAZ domain specifically binds siRNA with a 2 -nt 3 ' overhang, and the PIWI domain is similar to the RNase $\mathrm{H}$ domain and has RNA cleaving activity. The AGO proteins known as Slicers cleave target RNAs via the PIWI domain, while other AGO proteins recruit additional proteins into RISC to mediate translation repression or transcriptional gene silencing (TGS) [40].
Many VSRs have been shown to bind AGO proteins. The $2 \mathrm{~b}$ protein encoded by $\mathrm{CMV}$ was the first protein identified that binds AGO1 and AGO4 in vivo. AGO1 is the major effector in both miRNA-directed and virus-induced RNA silencing. The $2 \mathrm{~b}$ protein is co-localized with AGO1 both in the cytoplasm and nucleolus [41]. Bimolecular fluorescence complementation $(\mathrm{BiFC})$ and co-immunoprecipitation $(\mathrm{Co}-$ IP) assays have shown that $2 b$ interacts directly with the PAZ and PIWI domains of AGO1, leading to the inhibition of slicer activity of AGO1 [42]. 2b also interacts directly with AGO4 in the nucleolus [43]. AGO4 binds to 24-nt long repeat-associated siRNA (ra-siRNA) to participate in RNA-directed DNA methylation (RdDM) [17,44]. 2b competes with AGO4 for binding to 24-nt ra-siRNA and suppresses the DNA methylation that is mediated by AGO4 [43]. The influence of the inhibition by $2 b$ of RdDM on virus replication or spread remains to be investigated. 
Table 1 Summary of RNA binding activities of VSRs

\begin{tabular}{|c|c|c|c|}
\hline Virus & VSR & Characteristics of RNA binding & Reference \\
\hline Flock House virus & B2 & Binding dsRNA without size selection & [22] \\
\hline Influenza A virus & NS3 & Binding dsRNA without size selection & {$[45,46]$} \\
\hline Tomato aspermy virus & $2 b$ & Binding dsRNA without size selection & {$[26,27]$} \\
\hline Cucumber mosaic virus & $2 b$ & Binding dsRNA without size selection & [25] \\
\hline Rice stripe virus & NS3 & Binding dsRNA without size selection & {$[47]$} \\
\hline Pothos latent virus & $\mathrm{P} 14$ & Binding dsRNA without size selection & {$[24]$} \\
\hline Cymbidium ringspot virus & P19 & Preferential binding of $19 \mathrm{bp}$ dsRNA & [30] \\
\hline Tomato bushy stunt virus & P19 & Preferential binding of $19 \mathrm{bp}$ dsRNA & [29] \\
\hline Carnation Italian ringspot virus & P19 & Preferential binding of $19 \mathrm{bp}$ dsRNA & {$[28,32]$} \\
\hline Beet yellows virus & $\mathrm{P} 21$ & Preferential binding of siRNA/miRNA with 2 -nt $3^{\prime}$ overhang & [48] \\
\hline Peanut clump virus & P15 & Preferential binding of siRNA/miRNA with 2-nt $3^{\prime}$ overhang & [48] \\
\hline Barley stripe mosaic virus & $\gamma \mathrm{B}$ & Preferential binding of siRNA/miRNA with 2-nt $3^{\prime}$ overhang & [48] \\
\hline Tobacco mosaic virus & $\mathrm{P} 122$ & Preferential binding of siRNA/miRNA with 2 -nt $3^{\prime}$ overhang & [35] \\
\hline Cucumber vein yellowing virus & $\mathrm{P} 1 \mathrm{~b}$ & Binding dsRNA with size selection & [49] \\
\hline Rice hoja blanca virus & NS3 & Binding siRNA & {$[50]$} \\
\hline Rice dwarf virus & Pns 10 & Binding siRNA & [51] \\
\hline
\end{tabular}

Protein P0 from beet western yellows virus (BWYV) suppresses RNA silencing by interacting with AGO1 and promoting its degradation [52-55]. The fact that P0 does not inhibit the slicer activity of the programmed AGO1-siRNA/miRNA complex, but prevents de novo formation of an AGO1-containing high-molecular-weight (>670 kD) RISC-like complex in vivo, implies that $\mathrm{P} 0$ does not directly interact with AGO1 [56]. Early studies have shown that P0 interacts with the Arabidopsis S-phase kinase-related proteins (SKP) ASK1 and ASK2, which are components of SKP1/Cullin1/F-box/RBX1 (SCF) E3 ubiquitin ligase 3, via its F-box motif in the $\mathrm{N}$-terminal region [57]. However, the speculation that AGO1 is targeted by $\mathrm{P} 0$ for ubiquitination and proteasome-dependent degradation is not consistent with the fact that $\mathrm{P} 0$-mediated AGO1 degradation is insensitive to proteasome inhibitors [53]. Indeed, P0 enhances accumulation of K48-linked ubiquitinated proteins, including AGO1, but this activity is independent from the RNA silencing suppressor activity [56]. The relationship between the P0-SKP interaction and the RNA silencing suppressing function of $\mathrm{P} 0$ remains to be resolved.

Two recent studies have illustrated that P38 from TCV and P1 from sweet potato mild mottle virus (SPMMV) use a new strategy for binding AGO proteins $[58,59]$. These two VSRs mimic the endogenous GW/WG repetitive motif that is used for interacting with AGO proteins. GW/WG motifs have been identified in many silencing-related proteins, which include NRPD1a, the largest subunit of RNA polymerase $\mathrm{V}(\mathrm{Pol} \mathrm{V})$ in plants. These silencing-related proteins are recruited by AGO proteins into RISC or RdDM complexes in Drosophila [60-62]. These motifs serve as "AGO-hooks".

Two GW/WG motifs were identified at the N-terminal and C-terminal regions of $\mathrm{P} 38$. The interaction between
AGO1, AGO4 and the GW/WG motifs was detected in vitro, but P38 specifically interacted with AGO1 and not AGO4 in vivo [58]. There are several findings that indicate that P38 inhibits the activity of AGO1 to promote virus infection. Point mutations in the two GW repeats abolish both the interaction between P38 and AGO1 and the suppression of silencing by P38. TCV carrying the mutant P38 protein is partially rescued in agol-mutant A. thaliana plants [58]. Also, the accumulation of host miRNA that is dependent on AGO1 is reduced dramatically during TCV infection [58]. It is unknown whether P38 prevents AGO1 from being assembled into mature RISC or if it inhibits the silencing activity of programmed RISC. P38 also has the ability to bind siRNA and dsRNA [48]. It is not known whether the RNA binding activity of P38 plays a role in its binding of AGO1. Among the miRNAs whose accumulation is affected by TCV infection in a P38-dependent manner, that of miR162 is reduced, whereas the expression of its target gene $D C L 1$ is enhanced [63]. Also, the expression of DCLA and DCL3 is suppressed depending on the dramatically enhanced DCL1 levels, while DCL2 is not affected significantly [9,58], which explains why the 22-nt virus-derived siRNA is the major species present during TCV infection [7].

Protein P1 from SPMMV interacts with AGO1 through three GW repeats located at $\mathrm{N}$-terminal 383 amino acids. Point mutations in the GW repeats abolish both the binding to AGO1 and the VSR activity, indicating that AGO1 binding is essential for the VSR activity. P1 is co-fractionated with both AGO1 and host miRNAs in a high molecular weight $(>670 \mathrm{kD})$ RISC-like complex. Furthermore, P1 inhibits the silencing activity of mature RISC that has been preloaded with either a host miRNA or viral siRNAs. It has been speculated that high-affinity binding of AGO1 by P1 may outcompete an essential endogenous GW/WG-con- 
taining component of RISC or prevent recognition of target RNA via loading of complementary siRNA onto RISC [59].

P25 encoded by potato virus X (PVX) interacts with multiple members of the AGO family and promotes proteasome-dependent degradation of AGO1 [64]. P25 was one of the first identified VSRs and inhibits sense transgene or dsRNA-induced RNA silencing. P25 reduces the accumulation of both primary and secondary siRNA, but has no effect on the accumulation of endogenous miRNA and siRNA. Also, it inhibits systemic silencing, but does not inhibit the virus-induced gene silencing in locally-infected leaves $[65,66]$. Co-IP assays indicate that P25 interacts with AGO1, $\mathrm{AGO} 2, \mathrm{AGO} 3$ and AGO4 but not AGO5 or AGO7. The level of AGO1 is reduced by the expression of $\mathrm{P} 25$, and the degradation of AGO1 is blocked by the proteasome inhibitor MG132 [64]. Because P25 does not affect the endogenous miRNA or siRNA, it has been speculated that P25 does not interfere with the programmed RISC. P25 also promotes virus movement, which is dependent on VSR activity [67]. It is not currently known whether P25 inhibits the local movement of silencing signals by targeting AGO1 to promote virus movement.

Another report revealed a new strategy for the suppression of antiviral silencing that is possibly ubiquitous in plant-virus interactions. The expression of miR168, which controls the expression of AGO1, is enhanced during the infection of CymRSV in a manner that is dependent on the expression of P19. The down-regulated protein level of AGO1 results from the up-regulated miR168. This phenomenon is also observed in some other virus infections $[68,69]$. Because AGO1 is the major effector for miRNA-induced silencing, the deficiency of AGO1 could possibly lead to the misregulation of miRNA targets, resulting in disturbed gene expression which can in turn result in the development of virus symptoms [6]. For example, P38mediated inhibition of AGO1 modifies the cellular availability of the four Arabidopsis DCLs [58].

\subsection{VSRs interfere with secondary siRNA amplifica- tion}

In RVI, RDR1 and RDR6 recognize viral RNAs to produce secondary vsiRNAs, which play an essential role in antiviral defense [13-15].

Several lines of evidence have revealed that $2 b$ from CMV suppresses the function of RDR6. Graft assays have shown that $2 \mathrm{~b}$ blocks the long distance movement of systemic silencing signals and inhibits signal-inducing systemic silencing [70]. The 2b-defective mutant of CMV is defective in systemic infection of shoot apical meristems and leaf primordial [71]. The mutation of RDR6 promotes virus invasion of shoot apical meristems, and it has been shown that the spread of silencing signals between cells depends on RDR6 [72]. These results imply that $2 \mathrm{~b}$ may inhibit the function of RDR6 to promote systemic virus infection, which has been confirmed by a recent study: the 2b-defective CMV mutant restores virus infection in $r d r 6$ mutant plants, and the accumulation of vsiRNA is largely reduced [14]. This suggests that the $2 \mathrm{~b}$ protein specifically inhibits the amplification of RDR6-dependent secondary vsiRNA, which is essential for antiviral defense. However, whether $2 \mathrm{~b}$ binding to AGO1 and siRNA plays a role in its inhibition of secondary siRNA amplification remains to be revealed.

The V2 protein from tomato yellow curl leaf geminivirus-Israel (TYLCV-Is) may interfere with the amplification of secondary vsiRNA by interacting directly with SGS3 protein, which interacts with RDR6 and is involved in the RDR6-mediated silencing amplification pathway [73-77]. SGS3 protein consists of a Zinc-finger domain, a coiled-coiled domain and a plant-specific XS domain. SGS3 binds dsRNA with a 5' ssRNA overhang by the coiledcoiled and XS domains [78]. An in vitro study has shown that V2 competes with SGS3 protein for binding dsRNA with a 5' ssRNA overhang [78]. This finding might reveal a novel dsRNA intermediate formed during slicing, which is essential for SGS3/RDR6-dependent siRNA amplification in plants [79]. V2 protein may bind either SGS3 and/or the dsRNA intermediate to inhibit the dsRNA synthesis via the RDR6 pathway.

\subsection{VSRs target other related proteins of RNA silenc- ing}

P6 protein of cauliflower mosaic virus (CaMV) inhibits transgene and virus-induced gene silencing and suppresses the accumulation of endogenous trans-acting siRNA, but has no effect on the accumulation of miRNA [80]. P6 has two $\alpha$-importin-dependent nuclear localization signals. The VSR activity of P6 depends on its nuclear localization, implying that P6 suppresses the nuclear silencing components. P6 directly interacts with the dsRNA-binding protein DRB4, which is required for production of the DCL4-dependent 21-nt siRNA derived from endogenous TAS loci, exogenous transgenes and viral RNAs. The production of 21-nt siRNA by DCL4 is suppressed by P6, which is expressed from a stably integrated transgene [81].

\subsection{VSRs interfere with the epigenetic modification of the viral genome}

Small RNAs not only guide post-transcriptional gene silencing by RNA degradation or translational repression but also guide TGS by DNA methylation. The single-stranded DNA geminivirus genome is methylated during infection, and viruses with methylated genomes are defective in replication and infection [82-84]. However, many suppressors from the Geminivirus family interfere with the biochemical pathway of DNA methylation [85].

The AL2 protein encoded by tomato golden mosaic virus 
(TGMV) and the L2 protein encoded by beet curly top virus (BCTV) can reverse the established TGS. It reduces the host genome-wide cytosine methylation by interacting with and inactivating adenosine kinase (ADK) [86,87]. ADK is an enzyme that catalyzes the conversion of adenosine into AMP, which sustains the S-adenosyl methionine (SAM) cycle [88]. By inhibiting ADK, AL2 and L2 block the SAM cycle, interfering with the epigenetic modification of the viral genome. The finding that in vitro methylated TGMV cannot replicate in protoplasts suggests that methylation of the viral genome could be a valid strategy for combating geminivirus infections [83]. AL2 also functions as a transactivator. AL2 and L2 cause ectopic expression of endogenous $A$. thaliana loci that are silenced by methylation. At some loci, this ectopic expression is correlated with the inhibition of ADK by AL2 and L2, but at one exceptional locus ADK inhibition is insufficient, and TGS reversal requires the transcriptional activation domain of AL2. The AL2 mutant protein lacking the transcriptional activation domain can still suppress dsRNA-induced RNA silencing [89]. Further investigation is needed to determine whether some genes that are transactivated by AL2 are involved in the immunity of viruses. However, the silencing of suppression and activation of transcription by $\mathrm{AC} 2$ from mungbean yellow mosaic virus (MYMV) and African cassava mosaic virus (ACMV) are functionally related, suggesting that some of the AC2-induced genes may control RNA silencing through an endogenous network [90].

C2 protein encoded by beet severe curly top virus (BSCTV) interacts with S-adenosyl-methionine decarboxylase (SAMDC) and attenuates the $26 \mathrm{~S}$ proteasomemediated degradation of SAMDC1. SAMDC is an enzyme that catalyzes the conversion of SAM to decarboxylated S-adenosyl methionine (dcSAM). dcSAM shares similar radicals with SAM; therefore, dsSAM acts as a competitive inhibitor of SAM for methyltransferases. C2 suppresses de novo methylation of the host genome in $\mathrm{C} 2$ transgene plants. BSCTV carrying deficient C2 shows defects in infection and replication. $\mathrm{C} 2$ deficiency also causes enhanced methylation levels of the viral genome. Loss of function of SAMDC1 has a similar effect on virus infectivity and viral DNA accumulation as does BSCTV C2 deficiency. Overexpression of SAMDC1 suppresses the transgene-induced RNA silencing as well as $\mathrm{C} 2$. These results suggest that $\mathrm{C} 2$ protein prevents the viral genome from epigenetic modification, evading the host antiviral defense mechanism by attenuating the $26 \mathrm{~S}$ proteasome-mediated degradation of SAMDC1 [84].

Tomato yellow leaf curl China virus (TYLCCNV), belonging to the Old World begomoviruses, has the genome component DNA A. It acquires a satellite virus, TYLCCNB, whose genome is known as DNA $\beta$. DNA $\beta$ encodes a single protein, $\beta \mathrm{C} 1$, which acts as a VSR and pathogenicity factor. Co-inoculation of TYLCCNB with TYLCCNV, or expression of $\beta C 1$ protein, causes reduced methylation of both the genome of TYLCCNV and the host genome, and the reversal of TGS directed against a transgene and an endogenous locus. Yeast two-hybrid assays and BiFC assays show that $\beta C 1$ interacts with S-adenosyl-L-homocysteine hydrolase (SAHH), which is required to maintain the SAM cycle. The SAM-dependent methylases use SAM as a substrate to transfer methyl groups to produce S-adenosyl homocysteine (SAH). SAH is hydrolyzed to homocysteine (Hcy) and adenosine by SAHH, and Hcy is then recycled back to reproduce SAM. $\beta C 1$ inhibits $\mathrm{SAHH}$ activity in vitro, and the suppression of silencing of $\beta \mathrm{C} 1$ depends on the interaction with $\mathrm{SAHH}$. These results suggest that $\beta \mathrm{C} 1$ interferes with the host defense process of DNA methylation-mediated gene silencing by inhibiting the reproduction of SAM [82].

\section{Future directions}

\subsection{Multiple mechanisms used in the silencing of sup- pression}

Viral suppressors have been identified that target core components of RNA silencing, such as siRNA, AGO and DCL proteins. Some VSRs target multiple steps in the RNA silencing pathway. P38 binds AGO1 to interfere with the AGO1-dependent homeostatic network in which the expression of DCL4 and DCL3 is regulated [58]. One recent study revealed that the expression pattern of endogenous miRNA/miRNA* is changed greatly during the infection of rice stripe virus (RSV). The accumulation of rice miRNA*, but not the corresponding miRNA, as well as phased siRNA from a particular precursor, is enhanced during RSV infection. RSV infection also induces the expression of novel miRNA in a phased pattern from several conserved miRNA precursors [69]. This finding suggests the possibility that VSR may interfere with the gene regulation network that controls core elements of the RNA silencing pathway. The multiple mechanisms of many VSRs remain to be discovered, and there are still unknown elements in the RNA silencing pathway. For example, two kinds of RISCs with molecular weights of 150 and $670 \mathrm{kD}$ have recently been found in plants, but little is known about the mechanisms of assembly or the possible components of the two kinds of RISCs, which could also be potential targets of VSRs.

\subsection{The functions of VSRs in promoting the spread and replication of viruses}

VSRs help viruses escape host immunity by inhibiting RNA silencing. The mechanisms of the suppression of silencing are analyzed by transient expression systems or stable transgene expression systems. However, these expression systems do not reflect the natural expression during virus infection, because the natural expression of VSRs is restricted to virus-infected tissues and compartments and is 
limited by the virus life cycle. Only a few VSRs with roles in virus infection and replication have been identified. For example, the 2b-deletion mutant of CMV cannot infect wild type A. thaliana, but can infect $d c l 2 / 4, r d r l / 6$, agol, and ago2 mutant plants $[8,14,15]$. The silencing suppressor HC-Pro-deficient mutant of turnip mosaic virus (TuMV) also restores systemic infection in $d c l 2 / 4$ and $r d r l / 6$ mutant plants [13]. P19-deficient mutants of CymRSV accumulate only in the vascular bundles and exhibit defects in invading the surrounding tissues, suggesting that blocking the local movement of RNA silencing by P19 is essential for systemic virus infection [33]. Many VSRs have multiple functions in the virus life cycle. For example, the VSRs P25 and HC-Pro are also movement proteins for the virus. The separate analysis of their silencing suppressor activities can lead to misinterpretations; therefore, it is essential to analyze VSRs in their natural virus environments.

\subsection{VSRs and pathogenic determinants}

The antiviral and endogenous RNA silencing pathways share some common elements, which are the targets of VSRs. VSRs have been shown to interfere with the endogenous RNA silencing pathway, which is responsible for the regulation of development. The expression of many VSRs in transgenic plants leads to abnormal developmental phenotypes that mimic virus symptoms [91-93]. It is currently thought that VSRs are the pathogenic determinants that are largely responsible for virus-induced symptoms. However, the effect of transgenic expression of VSRs on endogenous silencing pathways does not reflect the effects of virus infection because the expression patterns of VSRs under these conditions are different. The 2b-deletion mutant of CMV and HC-Pro-deficient TuMV restore infection and develop symptoms in the $d c l 2 / 3 / 4$ triple mutant plants, suggesting that virus symptoms are not due to the expression of VSRs [8]. A recent report revealed that a siRNA derived from the Y satellite virus of CMV targets the host CHL1 gene, leading to the down-regulation of CHL1 gene and development of mosaic symptoms [94]. The mechanism of virus-induced symptoms requires further investigation.

\subsection{The effect of VSRs on systemic RNA silencing}

In plants, worms (Caenorhabditis elegans), insects (Tribolium) and Planaria, RNA silencing is associated with the production of a mobile signal that travels from cell-to-cell and also over long distances. The systemic RNA silencing in plants is established in two steps. Initially, the silencing signal moves into adjacent cells, referred to as local movement of RNA silencing. Second, the silencing signal moves between different organs. This step is referred to as systemic movement of RNA silencing and requires the silencing signal to be loaded into the vascular system, transported and then unloaded in the recipient tissue or cells [95]. Recent studies have identified that 21-nt and 24-nt siRNA are mobile and direct systemic RNA silencing [34,96,97].

As early as 1928, people observed that the development of ringspot symptoms on the upper leaves of tobacco plants infected with tobacco ringspot virus (TRSV) gradually declined, and the top leaves appeared perfectly normal. This "recovery" phenomenon is due to the inhibition of the virus by systemic RNA silencing, which is induced by the spreading of an RNA silencing signal from the inoculated leaves to the upper leaves. This finding suggests the essential role of systemic RNA silencing in antiviral defense. However, the mechanisms of VSRs in inhibiting systemic RNA silencing are not fully understood [95].

The VSRs that bind siRNA have been shown to interfere with the movement of the silencing signal. P19 blocks the local movement of the silencing signal by binding DCL4-dependent 21-nt siRNA, which is essential for systemic virus infection [34]. $2 \mathrm{~b}$ protein inhibits the systemic movement of RNA silencing either by binding dsRNA/siRNA or inhibiting the slicer activity of AGO1 $[25,42,70]$.

P25 protein also inhibits the systemic movement of RNA silencing, but the mechanism is unclear [65]. P25, which promotes the cell-to-cell movement of PVX, is associated with its silencing suppression activity [67]. It remains to be investigated whether the interference of P25 with the local movement of the RNA silencing signal promotes the cell-to-cell movement of virus. One recent study showed that virus-encoded multiple VSRs are responsible for suppressing different steps of RNA silencing. Of the two VSRs encoded by potato virus $\mathrm{M}$ (PVM), the cysteine-rich protein (CRP) enhances the accumulation of the viral genome in protoplasts and promotes the cell-to-cell movement of virus by inhibiting the local movement of RNA silencing. Another VSR, the triple gene block protein1 (TGBp1), does not have an effect on the accumulation of the viral genome in protoplast but is essential for systemic infection by the virus [98].

Many viruses are excluded in the shoot apical meristems. RDR6 participates in preventing meristem invasion by viruses [72]. The stable expression of Pns10 from rice dwarf virus leads to decreased expression of RDR6 and enhances virus infection and invasion of shoot apical meristems in Pns10-transgenic N. benthamiana plants [51,99]. These findings suggest that a mechanism of systemic RNA silencing in the meristem could involve RDR6-dependent secondary siRNA amplification. However, there is very little information about RNA silencing in the meristem. In the process involving RDR6, it is not clear whether the long ssRNA or dsRNA would be transported into meristem as the substrate of RDR6, or in which form RNA would move long distances in plants. Another important issue is to resolve which proteins, apart from RDR6, are involved in systemic RNA silencing. Apart from binding dsRNA/siRNA, the mechanism by which VSRs inhibit systemic RNA silencing remains to be discovered [95]. 
This work was supported by the National Basic Research Program of China (Grant No. 2012CB114004) and the National Natural Science Foundation of China (Grant Nos. 31030005 and 30910103904).

1 Ding S W. RNA-based antiviral immunity. Nat Rev Immunol, 2010, 10: 632-644

2 Chapman E J, Carrington J C. Specialization and evolution of endogenous small RNA pathways. Nat Rev Genet, 2007, 8: 884-896

3 Carthew R W, Sontheimer E J. Origins and mechanisms of miRNAs and siRNAs. Cell, 2009, 136: 642-655

4 Hutvagner G, Simard M J. Argonaute proteins: key players in RNA silencing. Nat Rev Mol Cell Biol, 2008, 9: 22-32

5 Voinnet O. Use, tolerance and avoidance of amplified RNA silencing by plants. Trends Plant Sci, 2008, 13: 317-328

6 Burgyan J, Havelda Z. Viral suppressors of RNA silencing. Trends Plant Sci, 2011, 16: 265-272

7 Deleris A, Gallego-Bartolome J, Bao J, et al. Hierarchical action and inhibition of plant Dicer-like proteins in antiviral defense. Science, 2006, 313: 68-71

8 Diaz-Pendon J A, Li F, Li W X, et al. Suppression of antiviral silencing by Cucumber mosaic virus $2 \mathrm{~b}$ protein in Arabidopsis is associated with drastically reduced accumulation of three classes of viral small interfering RNAs. Plant Cell, 2007, 19: 2053-2063

9 Qu F, Ye X, Morris T J. Arabidopsis DRB4, AGO1, AGO7, and RDR6 participate in a DCL4-initiated antiviral RNA silencing pathway negatively regulated by DCL1. Proc Natl Acad Sci USA, 2008, 105: 14732-14737

10 Llave C. Virus-derived small interfering RNAs at the core of plant-virus interactions. Trends Plant Sci, 2010, 15: 701-707

11 Qu F. Antiviral role of plant-encoded RNA-dependent RNA polymerases revisited with deep sequencing of small interfering RNAs of virus origin. Mol Plant Microbe In, 2010, 23: 1248-1252

12 Qi X, Bao F S, Xie Z. Small RNA deep sequencing reveals role for Arabidopsis thaliana RNA-dependent RNA polymerases in viral siRNA biogenesis. PLoS ONE, 2009, 4: e4971

13 Garcia-Ruiz H, Takeda A, Chapman E J, et al. Arabidopsis RNA-dependent RNA polymerases and dicer-like proteins in antiviral defense and small interfering RNA biogenesis during Turnip Mosaic Virus infection. Plant Cell, 2010, 22: 481-496

14 Wang X B, Jovel J, Udomporn P, et al. The 21-nucleotide, but not 22-nucleotide, viral secondary small interfering RNAs direct potent antiviral defense by two cooperative Argonautes in Arabidopsis thaliana. Plant Cell, 2011, 23: 1625-1638

15 Wang X B, Wu Q F, Ito T, et al. RNAi-mediated viral immunity requires amplification of virus-derived siRNAs in Arabidopsis thaliana. Proc Natl Acad Sci USA, 2010, 107: 484-489

16 Harvey J J W, Lewsey M G, Patel K, et al. An antiviral defense role of AGO2 in plants. PLoS ONE, 2011, 6: e14639

17 Mi S, Cai T, Hu Y, et al. Sorting of small RNAs into Arabidopsis argonaute complexes is directed by the $5^{\prime}$ terminal nucleotide. Cell, 2008, 133: 116-127

18 Vance V B, Anandalakshmi R, Pruss G J, et al. A viral suppressor of gene silencing in plants. Proc Natl Acad Sci USA, 1998, 95: 13079-13084

19 Baulcombe D C, Brigneti G, Voinnet O, et al. Viral pathogenicity determinants are suppressors of transgene silencing in Nicotiana benthamiana. EMBO J, 1998, 17: 6739-6746

20 Carrington J C, Kasschau K D. A counterdefensive strategy of plant viruses: Suppression of posttranscriptional gene silencing. Cell, 1998, 95: $461-470$

21 Ding S W, Wu Q F, Wang X B. Viral suppressors of RNA-Based viral Immunity: host targets. Cell Host Microbe, 2010, 8: 12-15

22 Williamson $\mathrm{J}$ R, Chao $\mathrm{J}$ A, Lee $\mathrm{J} \mathrm{H}$, et al. Dual modes of RNA-silencing suppression by flock house virus protein B2. Nat Struct Mol Biol, 2005, 12: 952-957

23 Ding S W, Aliyari R, Wu Q F, et al. Mechanism of induction and suppression of antiviral immunity directed by virus-derived small RNAs in Drosophila. Cell Host Microbe, 2008, 4: 387-397
24 Silhavy D, Merai Z, Kerenyi Z, et al. Aureusvirus P14 is an efficient RNA silencing suppressor that binds double-stranded RNAs without size specificity. J Virol, 2005, 79: 7217-7226

25 Masuta C, Goto K, Kobori T, et al. Characterization of silencing suppressor $2 \mathrm{~b}$ of cucumber mosaic virus based on examination of its small RNA-Binding abilities. Plant Cell Physiol, 2007, 48: 1050-1060

26 Chen J C H, Rashid U J, Hoffmann J, et al. Multiple targets for suppression of RNA interference by Tomato aspermy virus protein $2 \mathrm{~B}$. Biochemistry, 2008, 47: 12655-12657

27 AdamYuan Y, Chen H Y, Yang J, et al. Structural basis for RNA-silencing suppression by Tomato aspermy virus protein $2 \mathrm{~b}$. EMBO Rep, 2008, 9: 754-760

28 Hall T M T, Vargason J M, Szittya G, et al. Size selective recognition of siRNA by an RNA silencing suppressor. Cell, 2003, 115: 799-811

29 Patel D J, Ye K Q, Malinina L. Recognition of small interfering RNA by a viral suppressor of RNA silencing. Nature, 2003, 426: 874-878

30 Silhavy D, Molnar A, Lucioli A, et al. A viral protein suppresses RNA silencing and binds silencing-generated, 21- to 25-nucleotide double-stranded RNAs. EMBO J, 2002, 21: 3070-3080

31 Burgyan J, Lakatos L, Szittya G, et al. Molecular mechanism of RNA silencing suppression mediated by p19 protein of tombusviruses. EMBO J, 2004, 23: 876-884

32 Burgyan J, Lakatos L, Csorba T, et al. Small RNA binding is a common strategy to suppress RNA silencing by several viral suppressors. EMBO J, 2006, 25: 2768-2780

33 Havelda Z, Hornyik C, Crescenzi A, et al. In situ characterization of Cymbidium Ringspot tombusvirus infection-induced posttranscriptional gene silencing in Nicotiana benthamiana. J Virol, 2003, 77: 6082-6086

34 Dunoyer P, Schott G, Himber C, et al. Small RNA duplexes function as mobile silencing signals between plant cells. Science, 2010, 328: 912-916

35 Burgyan J, Csorba T, Bovi A, et al. The p122 subunit of Tobacco mosaic virus replicase is a potent silencing suppressor and compromises both small interfering RNA- and MicroRNA-mediated pathways. J Virol, 2007, 81: 11768-11780

36 Unrau P J, Ebhardt H A, Thi E P, et al. Extensive 3' modification of plant small RNAs is modulated by helper component-proteinase expression. Proc Natl Acad Sci USA, 2005, 102: 13398-13403

37 Burgyan J, Lozsa R, Csorba T, et al. Inhibition of 3' modification of small RNAs in virus-infected plants require spatial and temporal co-expression of small RNAs and viral silencing-suppressor proteins. Nucleic Acids Res, 2008, 36: 4099-4107

38 Valkonen J P T, Kreuze J F, Savenkov E I, et al. Viral class 1 RNase III involved in suppression of RNA silencing. J Virol, 2005, 79: 7227-7238

39 Valkonen J P T, Cuellar W J, Kreuze J F, et al. Elimination of antiviral defense by viral RNase III. Proc Natl Acad Sci USA, 2009, 106: 10354-10358

40 Meister G, Peters L. Argonaute proteins: Mediators of RNA silencing. Mol Cell, 2007, 26: 611-623

41 Canto T, Gonzalez I, Martinez L, et al. Cucumber Mosaic Virus 2b Protein subcellular targets and interactions: their significance to RNA silencing suppressor activity. Mol Plant Microbe In, 2010, 23: 294-303

42 Chua N H, Zhang X R, Yuan Y R, et al. Cucumber mosaic virus-encoded 2b suppressor inhibits Arabidopsis Argonaute1 cleavage activity to counter plant defense. Genes Dev, 2006, 20: 3255-3268

43 Hamera S, Song X G, Su L, et al. Cucumber mosaic virus suppressor $2 \mathrm{~b}$ binds to AGO4-related small RNAs and impairs AGO4 activities. Plant J, 2011, 69: 104-115

44 Pikaard C S, Pontes O, Li C F, et al. The Arabidopsis chromatin-modifying nuclear siRNA pathway involves a nucleolar RNA processing center. Cell, 2006, 126: 79-92

45 Prins M, Bucher E, Hemmes H, et al. The influenza A virus NS1 protein binds small interfering RNAs and suppresses RNA silencing in plants. J Gen Virol, 2004, 85: 983-991

46 Ding $\mathrm{S} \mathrm{W}, \mathrm{Li} \mathrm{W} \mathrm{X,} \mathrm{Li} \mathrm{H} \mathrm{W,} \mathrm{et} \mathrm{al.} \mathrm{Interferon} \mathrm{antagonist} \mathrm{proteins} \mathrm{of}$ 
influenza and vaccinia viruses are suppressors of RNA silencing. Proc Natl Acad Sci USA, 2004, 101: 1350-1355

47 Ye K Q, Shen M, Xu Y, et al. Size-independent and noncooperative recognition of dsRNA by the Rice stripe virus RNA silencing suppressor NS3. J Mol Biol, 2010, 404: 665-679

48 Silhavy D, Merai Z, Kerenyi Z, et al. Double-stranded RNA binding may be a general plant RNA viral strategy to suppress RNA silencing. J Virol, 2006, 80: 5747-5756

49 Valli A, Oliveros J C, Molnar A, et al. The specific binding to 21-nt double-stranded RNAs is crucial for the anti-silencing activity of Cucumber vein yellowing virus P1b and perturbs endogenous small RNA populations. RNA, 2011, 17: 1148-1158

50 Lakatos L, Hemmes H, Goldbach R, et al. The NS3 protein of Rice hoja blanca tenuivirus suppresses RNA silencing in plant and insect hosts by efficiently binding both siRNAs and miRNAs. RNA, 2007, 13: 1079-1089

51 Ren B, Guo Y Y, Gao F, et al. Multiple functions of Rice dwarf phytoreovirus Pns 10 in suppressing systemic RNA silencing. J Virol, 2010, 84: 12914-12923

52 Ziegler-Graff V, Pfeffer S, Dunoyer P, et al. P0 of beet western yellows virus is a suppressor of posttranscriptional gene silencing. J Virol, 2002, 76: 6815-6824

53 Baumberger N, Tsai C $\mathrm{H}$, Lie $\mathrm{M}$, et al. The Polerovirus silencing suppressor P0 targets ARGONAUTE proteins for degradation. Curr Biol, 2007, 17: 1609-1614

54 Bortolamiol D, Pazhouhandeh M, Marrocco K, et al. The Polerovirus $\mathrm{F}$ box protein $\mathrm{P} 0$ targets ARGONAUTE1 to suppress RNA silencing. Curr Biol, 2007, 17: 1615-1621

55 Mirkov T E, Mangwende T, Wang M L, et al. The P0 gene of Sugarcane yellow leaf virus encodes an RNA silencing suppressor with unique activities. Virology, 2009, 384: 38-50

56 Burgyan J, Csorba T, Lozsa R, et al. Polerovirus protein P0 prevents the assembly of small RNA-containing RISC complexes and leads to degradation of ARGONAUTE1. Plant J, 2010, 62: 463-472

57 Ziegler-Graff V, Pazhouhandeh M, Dieterle M, et al. F-box-like domain in the Polerovirus protein $\mathrm{P} 0$ is required for silencing suppressor function. Proc Natl Acad Sci USA, 2006, 103: 1994-1999

58 Azevedo J, Garcia D, Pontier D, et al. Argonaute quenching and global changes in Dicer homeostasis caused by a pathogen-encoded GW repeat protein. Genes Dev, 2010, 24: 904-915

59 Giner A, Lakatos L, Garcia-Chapa M, et al. Viral protein inhibits RISC activity by Argonaute binding through conserved WG/GW motifs. PLoS Pathog, 2010, 6: e1000996

60 Ladurner A G, Till S. RNA Pol IV plays catch with argonaute 4. Cell, 2007, 131: 643-645

61 Lagrange T, El-Shami M, Pontier D, et al. Reiterated WG/GW motifs form functionally and evolutionarily conserved ARGONAUTEbinding platforms in RNAi-related components. Genes Dev, 2007, 21: 2539-2544

62 Karlowski W M, Zielezinski A, Carrere J, et al. Genome-wide computational identification of WG/GW Argonaute-binding proteins in Arabidopsis. Nucleic Acids Res, 2010, 38: 4231-4245

63 Carrington J C, Xie Z X, Kasschau K D. Negative feedback regulation of Dicer-like1 in Arabidopsis by microRNA-guided mRNA degradation. Curr Biol, 2003, 13: 784-789

64 Chiu M H, Chen I H, Baulcombe D C, et al. The silencing suppressor P25 of Potato virus $X$ interacts with Argonaute1 and mediates its degradation through the proteasome pathway. Mol Plant Pathol, 2010, 11: 641-649

65 Voinnet O, Lederer C, Baulcombe D C. A viral movement protein prevents spread of the gene silencing signal in Nicotiana benthamiana. Cell, 2000, 103: 157-167

66 Moissiard G, Parizotto E A, Himber C, et al. Transitivity in Arabidopsis can be primed, requires the redundant action of the antiviral Dicer-like 4 and Dicer-like 2, and is compromised by viral-encoded suppressor proteins. RNA, 2007, 13: 1268-1278

67 Bayne E H, Rakitina D V, Morozov S Y, et al. Cell-to-cell movement of Potato Potexvirus $\mathrm{X}$ is dependent on suppression of RNA silencing. Plant J, 2005, 44: 471-482
68 Havelda Z, Varallyay E, Valoczi A, et al. Plant virus-mediated induction of miR168 is associated with repression of ARGONAUTE1 accumulation. EMBO J, 2010, 29: 3507-3519

69 Du P, Wu J G, Zhang J Y, et al. Viral infection induces expression of novel phased microRNAs from conserved cellular microRNA precursors. PLoS Pathog, 2011, 7: e1002176

70 Ding S W, Guo H S. A viral protein inhibits the long range signaling activity of the gene silencing signal. EMBO J, 2002, 21: 398-407

71 Mochizuki T, Sunpapao A, Nakai T, et al. The 2b protein of cucumber mosaic virus is essential for viral infection of the shoot apical meristem and for efficient invasion of leaf primordia in infected tobacco plants. J Gen Virol, 2009, 90: 3015-3021

72 Schwach F, Vaistij F E, Jones L, et al. An RNA-dependent RNA polymerase prevents meristem invasion by potato virus $X$ and is required for the activity but not the production of a systemic silencing signal. Plant Physiol, 2005, 138: 1842-1852

73 Gafni Y, Zrachya A, Glick E, et al. Suppressor of RNA silencing encoded by Tomato yellow leaf curl virus-Israel. Virology, 2007, 358: $159-165$

74 Gafni Y, Glick E, Zrachya A, et al. Interaction with host SGS3 is required for suppression of RNA silencing by tomato yellow leaf curl virus V2 protein. Proc Natl Acad Sci USA, 2008, 105: 157-161

75 Mourrain P, Beclin C, Elmayan T, et al. Arabidopsis SGS2 and SGS3 genes are required for posttranscriptional gene silencing and natural virus resistance. Cell, 2000, 101: 533-542

76 Peragine A, Yoshikawa M, Wu G, et al. SGS3 and SGS2/SDE1/ RDR6 are required for juvenile development and the production of trans-acting siRNAs in Arabidopsis. Genes Dev, 2004, 18: 2368-2679

77 Watanabe Y, Kumakura N, Takeda A, et al. SGS3 and RDR6 interact and colocalize in cytoplasmic SGS3/RDR6-bodies. FEBS Lett, 2009, 583: $1261-1266$

78 Doudna J A, Fukunaga R. dsRNA with 5' overhangs contributes to endogenous and antiviral RNA silencing pathways in plants. EMBO J, 2009, 28: 545-555

79 Elkashef S, Ding S W. Possible new RNA intermediate in RNA silencing. Nat Chem Biol, 2009, 5: 278-279

80 Milner J J, Love A J, Laird J, et al. Cauliflower mosaic virus protein P6 is a suppressor of RNA silencing. J Gen Virol, 2007, 88: 3439-3444

81 Keller M, Haas G, Azevedo J, et al. Nuclear import of CaMV P6 is required for infection and suppression of the RNA silencing factor DRB4. EMBO J, 2008, 27: 2102-2112

82 Yang X L, Xie Y, Raja P, et al. Suppression of methylation-mediated transcriptional gene silencing by $\beta \mathrm{C} 1-\mathrm{SAHH}$ protein interaction during Geminivirus-Betasatellite Infection. PLoS Pathog, 2011, 7: e1002329

83 Brough C L, Gardiner W E, Inamdar N M, et al. DNA methylation inhibits propagation of Tomato golden mosaic-virus DNA in transfected protoplasts. Plant Mol Biol, 1992, 18: 703-712

84 Zhang Z H, Chen H, Huang X H, et al. BSCTV C2 attenuates the degradation of SAMDC1 to suppress DNA methylation-mediated gene silencing in Arabidopsis. Plant Cell, 2011, 23: 273-288

85 Bisaro D M. Silencing suppression by geminivirus proteins. Virology, 2006, 344: 158-168

86 Bisaro D M, Wang H, Buckley K J, et al. Adenosine kinase inhibition and suppression of RNA silencing by geminivirus AL2 and L2 proteins. J Virol, 2005, 79: 7410-7418

87 Bisaro D M, Wang H, Hao L H, et al. Adenosine kinase is inactivated by geminivirus AL2 and L2 proteins. Plant Cell, 2003, 15: 3020-3032

88 Moffatt B A, Stevens Y Y, Allen M S, et al. Adenosine kinase deficiency is associated with developmental abnormalities and reduced transmethylation. Plant Physiol, 2002, 128: 812-821

89 Bisaro D M, Buchmann R C, Asad S, et al. Geminivirus AL2 and L2 proteins suppress transcriptional gene silencing and cause genomewide reductions in cytosine methylation. J Virol, 2009, 83: 5005-5013

90 Pooggin M A, Trinks D, Rajeswaran R, et al. Suppression of RNA 
silencing by a geminivirus nuclear protein, AC2, correlates with Transactivation of host genes. J Virol, 2005, 79: 2517-2527

91 Chapman E J, Prokhnevsky A I, Gopinath K, et al. Viral RNA silencing suppressors inhibit the microRNA pathway at an intermediate step. Genes Dev, 2004, 18: 1179-1186

92 Voinnet O, Dunoyer P, Lecellier C H, et al. Probing the microRNA and small interfering RNA pathways with virus-encoded suppressors of RNA silencing. Plant Cell, 2004, 16: 1235-1250

93 Carrington J C, Kasschau K D, Xie Z X, et al. P1/HC-Pro, a viral suppressor of RNA silencing, interferes with Arabidopsis development and miRNA function. Dev Cell, 2003, 4: 205-217

94 Wang M B, Smith N A, Eamens A L. Viral Small Interfering RNAs target host genes to mediate disease symptoms in plants. PLoS Pathog, 2011, 7: e1002022
95 Melnyk C W, Molnar A, Baulcombe D C. Intercellular and systemic movement of RNA silencing signals. EMBO J, 2011, 30: 3553-3563

96 Molnar A, Melnyk C W, Bassett A, et al. Small silencing RNAs in plants are mobile and direct epigenetic modification in recipient cells. Science, 2010, 328: 872-875

97 Dunoyer P, Brosnan C A, Schott G, et al. An endogenous, systemic RNAi pathway in plants. EMBO J, 2010

98 Senshu H, Yamaji Y, Minato N, et al. A dual strategy for the suppression of host antiviral silencing: two distinct suppressors for viral replication and viral movement encoded by Potato Virus M. J Virol, 2011, 85: 10269-10278

99 Cao X S, Zhou P, Zhang X M, et al. Identification of an RNA silencing suppressor from a plant double-stranded RNA virus. J Virol, 2005, 79: $13018-13027$

Open Access This article is distributed under the terms of the Creative Commons Attribution License which permits any use, distribution, and reproduction in any medium, provided the original author(s) and source are credited. 